\title{
Modelado de sistemas complejos mediante simulación basada en agentes y mediante dinámica de sistemas
}

\author{
Luis R. IZQUierdo, José M. GALÁN, José I. SANTOS y \\ RICARDO DEL OLMO \\ Universidad de Burgos \\ lrizquierdo@ubu.es \\ jmgalan@ubu.es \\ jisantos@ubu.es \\ rdelolmo@ubu.es
}

Recibido: 05.03.2008

Aceptado: 04.11.2008

\section{INTRODUCCIÓN}

En las últimas décadas, debido en gran parte al desarrollo del microprocesador, han aparecido innovadoras técnicas de modelado de sistemas complejos - y en particular de sistemas sociales - que hacen uso de la nueva tecnología disponible. Dos de estas técnicas son la simulación basada en agentes y la dinámica de sistemas.

Tanto la simulación basada en agentes como la dinámica de sistemas tienen el potencial de complementar modelos no formales (normalmente verbales) de sistemas complejos y modelos matemáticos más abstractos. A menudo la representación verbal de sistemas complejos carece del rigor formal necesario para valorar su coherencia lógica y para generalizar a partir de ellos, mientras que los modelos matemáticos suelen ser menos realistas debido a las fuertes restricciones impuestas por las hipótesis simplificadoras que son necesarias para poder resolverlos. Usando técnicas de modelado computacional como la simulación basada en agentes y la dinámica de sistemas tenemos el potencial de construir modelos que combinan la riqueza descriptiva de los modelos verbales con el rigor formal de los modelos matemáticos más abstractos.

Para poder aprovechar al máximo el potencial que cada una de estas técnicas nos ofrece, debemos ser conscientes de las diferencias que existen entre ellas y de aquellas características que las separan de los métodos tradicionales de mo- 
delado científico. De esta forma podremos valorar cuáles son sus ventajas y sus limitaciones.

En este artículo se realiza una comparación de la simulación basada en agentes y de la dinámica de sistemas como metodologías de modelado científico de sistemas complejos. La estructura del artículo es la siguiente: la segunda sección presenta una breve introducción al proceso de modelado científico. A continuación se recogen los principios esquematizados en la sección segunda y se particularizan para el caso del modelado computacional de sistemas complejos. La cuarta y quinta sección describen, respectivamente, la simulación basada en agentes y la dinámica de sistemas. Posteriormente se destacan las diferencias existentes entre las dos metodologías tomando como esquema general el proceso de modelado científico descrito en las primeras secciones. Finalmente se presentan las conclusiones.

\section{UNA ABSTRACCIÓN DEL PROCESO DE MODELADO CIENTÍFICO}

\section{Primera aproximación al proceso de modelado científico}

Varios filósofos (por ejemplo, Hesse (1963) y Hughes (1997)) que han estudiado la metodología científica tradicional han propuesto el mismo esquema general del proceso de modelado. Según estos autores, los modelos científicos se construyen para desarrollar procesos de inferencia sobre ciertos aspectos de sistemas reales previamente observados. Es mediante estos procesos de inferencia, mediante la construcción y el uso de modelos científicos, como mejoramos nuestro entendimiento de los sistemas reales observados. De hecho, man-

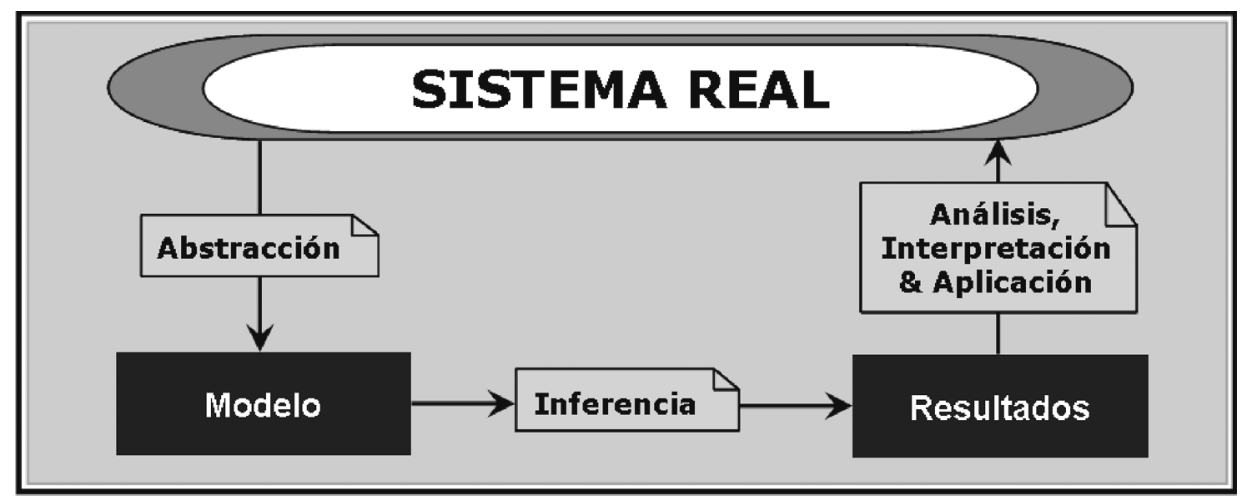

Fig. 1. Esquema general del proceso de modelado científico. La figura muestra un proceso secuencial por claridad pero, como se explica en el texto, el proceso de modelado contiene en general varios bucles de retroalimentación. 
teniendo el concepto de modelo suficientemente amplio, nosotros no vemos otra forma de mejorar nuestro conocimiento del mundo en que vivimos si no es a través de la creación de modelos (no necesariamente formales). El proceso de creación y uso de un modelo se muestra esquematizado en la figura 1.

Es posible que algunos lectores encuentren la siguiente interpretación del proceso de modelado científico excesivamente general y simplista. Realizamos esta simplificación con la intención de evitar introducir en nuestras explicaciones mayor complejidad de la estrictamente necesaria para lograr nuestros propósitos, i.e. discernir de forma clara la esencia que distingue a dos enfoques de modelado de sistemas complejos: la simulación basada en agentes y la dinámica de sistemas. Para un estudio más completo sobre la noción de modelo en diferentes disciplinas científicas, referimos al lector al artículo de Armatte (2006) que, en particular, contempla explícitamente el modelado computacional de sistemas complejos mediante dinámica de sistemas.

Nosotros entendemos que los modelos se construyen mediante procesos de abstracción con el objetivo de facilitar la comprensión de ciertos aspectos de un determinado sistema real. De este modo, parte de la complejidad del sistema real se pierde — de forma intencionada — en el proceso de creación de un modelo. El proceso de abstracción para crear un modelo comienza con la observación del sistema real (incluyendo, a menudo, la recogida de datos), y termina con el diseño del modelo. Diseñar un modelo requiere destilar la esencia del sistema real, lo cual implica prescindir de aquellos aspectos que no consideramos fundamentales para nuestros propósitos.

Como ejemplo, piense que a la hora de estudiar la caída libre de un objeto en la atmósfera terrestre, un buen modelador se abstraería del color de dicho objeto. La abstracción obtenida, i.e. el modelo, es normalmente notablemente más simple que la realidad, y es esta reducción en complejidad lo que nos permite usar el modelo para desarrollar procesos de inferencia que no podríamos acometer sin él. Así, nuestra motivación para llevar a cabo este proceso de abstracción (el cual implica una cierta pérdida de realismo) es que el modelo nos permitirá inferir algún tipo de conocimiento sobre el sistema real que no resultaba evidente antes de construir y usar el modelo.

Una vez diseñado el modelo, podremos llevar a cabo procesos de inferencia deductivos para averiguar las implicaciones lógicas que se derivan de las premisas que definen el modelo. Estas implicaciones lógicas darán lugar a una serie de resultados sobre el modelo, los cuales podrán ser analizados, interpretados y aplicados al sistema real objeto de estudio (ver fig. 1).

El término aplicación no debe entenderse necesariamente en su sentido práctico más estricto. La etapa de aplicación representa el retorno al sistema real desde el ámbito abstracto en el que reside nuestro modelo. Este viaje de vuelta consiste simplemente en relacionar las inferencias obtenidas usando el modelo — una vez analizadas e interpretadas - con el sistema real. Esta aplicación del conocimiento obtenido con el modelo puede ser concreta y específica (como p. ej. una predicción numérica en el campo de la física), pero también puede ser 
abstracta y general - y no por ello menos útil- (como p. ej. una explicación tentativa y aproximada de los resultados de unas elecciones).

En general, las conclusiones a las que podamos llegar usando un modelo no describirán con rigurosa exactitud lo que ocurre en el sistema real (especialmente en sistemas con un alto grado de incertidumbre) pero, cuando menos, aportarán un conocimiento significativamente mejor que el que obtendríamos sin aplicar modelo alguno.

El valor de un modelo no se limita necesariamente al análisis del sistema real para el cual el modelo fue diseñado. El conocimiento adquirido mediante el estudio de un determinado modelo puede trascender a otros sistemas reales cuya esencia quede capturada por la misma abstracción, incluso aunque el modelo no se desarrollase inicialmente con estos otros sistemas reales en mente. Como ejemplo, considere el estudio de cómo un teléfono móvil se aproxima al suelo cuando uno lo deja caer desde cierta altura. El modelo de gravedad Newtoniano nos puede ayudar considerablemente a entender esta situación, pese a que obviamente Newton no tenía en mente la caída libre de teléfonos móviles cuando desarrolló su teoría.

En resumen, un modelo será útil en la medida en que capture la esencia de la situación real objeto de estudio, facilite el desarrollo de procesos de inferencia que no se podrían llevar a cabo sin el modelo, y proporcione conocimientos que puedan ser transferidos a diversas situaciones.

\section{Retroalimentación en el proceso de modelado}

El proceso de modelado secuencial esquematizado en la figura 1 es una primera aproximación, una simplificación didáctica que conviene matizar. Resulta claro que los resultados obtenidos con un modelo - y el contraste de estos resultados con el sistema real- pueden poner en evidencia aspectos mejorables de las premisas que definen el modelo. Por ello, y sin ánimo de complicar excesivamente esta exposición, sí que consideramos conveniente resaltar que el proceso de modelado es en realidad raramente unidireccional. En la práctica, el proceso de modelado es en general muy dinámico y no lineal.

A menudo el modelador diseña un primer modelo con el cual obtiene unos resultados iniciales. A la luz de los resultados obtenidos, el modelador suele volver a las etapas iniciales del proceso de modelado para perfeccionar el modelo mediante la revisión de algunas de sus premisas. Es posible que los resultados obtenidos inicialmente se considerasen insatisfactorios (a) porque no parecieran derivarse lógicamente de las premisas del modelo (proceso de verificación) o (b) porque - aún siendo lógicamente correctos- difirieran excesivamente de los resultados observados en el sistema real que se está modelando (proceso de validación). A continuación explicamos brevemente estos dos procesos: 
- Verificación: El proceso de verificación ${ }^{1}$ consiste en comprobar que el modelo desarrollado cumple con los requisitos de diseño auto-impuestos por el modelador (Kleijnen 1995; Sargent 2003). De forma intuitiva, verificar un modelo consiste en asegurarse de que el modelo es lo que su diseñador piensa que es. El proceso de verificación es especialmente relevante en el desarrollo de modelos formales ${ }^{2}$, en los que el proceso de implementación del modelo (en un lenguaje formal) y la derivación lógica de resultados (mediante inferencia deductiva) no están exentos de errores.

Como ejemplo, la primera implementación de un modelo computacional a menudo produce resultados que son incoherentes con las premisas del modelo que el programador pretendía implementar. Un ejemplo sería observar que una variable en el modelo computacional toma valores fuera del rango admisible (p. ej. una variable porcentual que toma valores negativos). Esta observación sería una indicación clara de que ha habido un error en la programación y, por lo tanto, el código debería revisarse. Este tipo de revisión, que no hace referencia alguna al mundo real, formaría parte del proceso de verificación.

- Validación: En contraste con la verificación, la validación científica sí que hace referencia explícita al sistema real que se está modelando. Validar un modelo consiste en valorar su utilidad dentro del contexto de aplicación, de acuerdo con los criterios para los cuales el modelo fue diseñado (Kleijnen 1995; Sargent 2003) ${ }^{3}$. En el ámbito científico, el propósito de construir modelos (y el criterio por el que se valoran) suele ser avanzar nuestro conocimiento sobre un determinado sistema real. Así, por ejemplo, si nuestro objetivo es representar de forma fiel un proceso social dado, entonces un modelo será tanto más válido cuanto mejor capture la esencia de su referente empírico. Esto podría valorarse estudiando la medida en que los resultados obtenidos con el modelo se ajustan a los observados en el referente empírico (Moss et al. 1997).

De una manera un tanto informal, podríamos decir que verificar consiste en valorar si el modelo que tenemos es correcto, mientras que validar consiste en estudiar si tenemos el modelo correcto.

${ }^{1}$ El proceso de verificación también se denomina «validación interna» (p. ej. por Taylor (1983), Axelrod (1997a), Drogoul et al. (2003), y Sansores y Pavón (2005)).

${ }^{2}$ Usamos el término 'modelo formal' para denotar un sistema formal interpretado. Un sistema formal está compuesto por un lenguaje formal (que contiene un alfabeto y una gramática) y por un aparato deductivo (que contiene axiomas y reglas de inferencia). En un sistema formal las proposiciones carecen de significado intrínseco. Cuando se asigna un cierto significado a cada una de las proposiciones derivadas en el sistema formal decimos que el sistema formal se ha interpretado. Al sistema formal interpretado lo denominamos 'modelo formal'.

${ }^{3}$ El lector puede encontrar una revisión epistémica del proceso de validación en simulación en Kleindorfer et al. (1998), y una discusión dentro del contexto específico de la simulación basada en agentes en Windrum et al. (2007) y en Moss (2008).

EMPIRIA. Revista de Metodología de Ciencias Sociales. N. ${ }^{\circ}$ 16, julio-diciembre, 2008, pp. 85-112. ISSN: 1139-5737 


\section{Nuevas formas de implementar y analizar modelos formales}

Tradicionalmente, en el dominio de las ciencias naturales, los modelos científicos se han formalizado en ecuaciones matemáticas y, por lo tanto, las condiciones iniciales y las predicciones derivadas de éstas han tenido en general un formato numérico. Bajo este esquema, el trabajo de los científicos naturalistas ha consistido en establecer las relaciones entre el sistema real y el modelo matemático abstraído (en ambos sentidos, i.e. tanto en la recogida de datos y en el diseño del modelo, como en la interpretación y aplicación de los resultados obtenidos con él), mientras que los matemáticos se han venido preocupando de desarrollar y perfeccionar los procesos de inferencia y de análisis de resultados. En otros dominios, como en el de las ciencias sociales, el proceso de modelado se ha llevado a cabo, en numerosas ocasiones, creando modelos verbales. Este tipo de modelos no formales ha sacrificado, al menos hasta cierto punto, la precisión y la coherencia lógica de los modelos matemáticos para conseguir a cambio mayor riqueza descriptiva, flexibilidad y realismo.

Actualmente tenemos a nuestra disposición nuevas y potentes herramientas de inferencia lógica: los ordenadores. Con el nacimiento y desarrollo del microprocesador han aparecido nuevos enfoques de modelado científico que hacen uso de la simulación computacional como proceso inferencial. En las simulaciones computacionales, el modelo se codifica en un lenguaje de programación formal, y la inferencia se lleva a cabo ejecutando el programa informático desarrollado. Así, el actual desarrollo de la informática nos permite crear y estudiar mediante simulación computacional modelos formales que, debido a su complejidad, venían siendo intratables matemáticamente.

En este trabajo se comparan dos técnicas de modelado científico computacional: la simulación basada en agentes y la dinámica de sistemas. Como veremos a continuación, estas dos técnicas son capaces de avanzar nuestro conocimiento científico mediante la creación, el uso y el análisis de modelos formales de sistemas complejos.

\section{MODELADO COMPUTACIONAL DE SISTEMAS COMPLEJOS}

El proceso de modelado computacional de sistemas complejos se desvía un tanto del proceso de modelado tradicional explicado en la sección anterior. Antes de discutir estas diferencias, conviene que expliquemos lo que entendemos por sistemas complejos, puesto que la notación en la literatura no es, ni mucho menos, uniforme. Presentamos las siguientes características de los sistemas complejos con el mero propósito de delimitar el dominio de nuestras explicaciones posteriores, sin pretender calificar nuestra notación como la correcta, puesto que hay muchas otras igualmente válidas. En cualquier caso, repasando las principales propiedades que caracterizan a un sistema complejo resultará evidente que la mayoría de los sistemas sociales son claramente complejos. 


\section{Sistemas complejos}

Básicamente, un sistema puede definirse como un «conjunto de elementos en interacción» (Bertalanffy, 1968). Los sistemas complejos (p. ej. organismos pluricelulares, colonias de hormigas, ecosistemas, economías, sociedades...) están caracterizados por tener una estructura compuesta por varios niveles. En estos sistemas complejos (Vicsek, 2002; Gilbert, 2004):

- Los componentes de niveles jerárquicos inferiores suelen mostrar un grado de autonomía significativo.

- El comportamiento del sistema surge a partir de la auto-organización de sus componentes, sin que esta organización esté controlada ni dirigida por ningún ente exterior al sistema.

- Los componentes básicos de estos sistemas complejos (células, hormigas, individuos, poblaciones, empresas...) perciben su entorno y responden a cambios en él de forma potencialmente diferente.

Por si esto fuera poco, muchos sistemas complejos son también adaptativos. En estos sistemas adaptativos (organismos, ecosistemas, economías, sociedades...), el comportamiento de los componentes básicos del sistema puede evolucionar en el tiempo, dando lugar a una cierta capacidad de respuesta frente a cambios en el entorno por medio de mecanismos de:

- Aprendizaje a escala individual, y/o

- selección y reemplazo (lo cual da lugar a un aprendizaje a escala poblacional).

Todas estas características hacen que el proceso de modelado formal de sistemas complejos difiera sustancialmente del de otros sistemas más simples. En particular, su naturaleza descentralizada, la presencia de bucles de causalidad y retroalimentación no lineales, y el hecho de contener varias unidades más o menos autónomas, que pueden interaccionar, evolucionar, y adaptar su comportamiento a cambios en el entorno, implica que en la mayoría de los casos es muy difícil — si no imposible - conseguir un modelo que pueda describir el sistema complejo adecuadamente y que además sea resoluble matemáticamente.

\section{La simulación computacional como herramienta de análisis de modelos formales}

Hasta hace pocas décadas, no poder resolver matemáticamente un modelo formal suponía una desventaja importante, puesto que carecíamos de otras herramientas con las que deducir las implicaciones lógicas que de él se derivaban. Esto cambió radicalmente con la invención y el desarrollo del ordenador. Hoy en 
día, los ordenadores nos permiten explorar y analizar modelos formales que no podemos resolver matemáticamente. De esta forma, usando las nuevas tecnologías podemos implementar y analizar rigurosamente el comportamiento de modelos formales de sistemas complejos - algo inviable hasta hace poco- .

Un modelo que está implementado y que puede ejecutarse en un ordenador es un modelo necesariamente formal (Suber, 2007) y, por lo tanto, no difiere sustancialmente de un modelo matemático tradicional. Tanto los modelos computacionales como los modelos matemáticos son modelos formales; la única diferencia fundamental es que están expresados en lenguajes diferentes. En particular, todo modelo computacional puede expresarse en lenguaje matemático como un conjunto de ecuaciones - potencialmente muy difícil de resolver- (ver p. ej. Epstein, 2006; Leombruni \& Richiardi, 2005). En esencia, un modelo formal (sea éste computacional o matemático) puede verse como un conjunto de proposiciones que se admiten como ciertas (axiomas) más un conjunto de reglas de inferencia que pueden usarse para deducir nuevas proposiciones a partir de los axiomas y de proposiciones inferidas previamente.

Como ejemplo, uno podría escribir el programa computacional $\langle y=2 \cdot x »($ regla) y aplicarlo a las condiciones iniciales $\langle x=10 »$ (axioma) para obtener el resultado $« y=20 »$. El resultado $(y=20)$, que está unívoca e inequívocamente determinado por las condiciones iniciales $(x=10)$ y el conjunto de reglas que definen el modelo $(y=2 \cdot x)$ puede verse como un teorema matemático obtenido mediante deducción lógica ( $\{x=10 ; y=2 \cdot x\} \square y=20$ ). Naturalmente, no hay razón por la que las condiciones iniciales, las reglas o los resultados deban ser necesariamente numéricos; podrían ser, por ejemplo, cadenas de caracteres con significado en un determinado lenguaje verbal.

En el caso general, la inferencia obtenida al ejecutar un modelo computacional tiene la siguiente forma: Los resultados obtenidos al ejecutar la simulación computacional se derivan por deducción (y, por lo tanto, con necesidad lógica) de aplicar las reglas algorítmicas que definen el modelo al conjunto de condiciones iniciales con las que el modelo se ha parametrizado. De esta forma, independientemente de su mayor o menor complejidad interna, una simulación computacional constituye un teorema de suficiencia perfectamente válido (ver p. ej. Axtell, 2000).

Resulta útil pensar que uno siempre podría aplicar las mismas reglas de inferencia codificadas en el modelo computacional para obtener - por deducción lógica - los mismos resultados dadas las mismas condiciones iniciales. Esta afirmación, como pensamiento, resulta útil, pero a la hora de obtener resultados es mucho más eficiente, rápido y fiable dejar que un ordenador haga el trabajo por nosotros. Los ordenadores son máquinas de inferencia capaces de llevar a cabo procesos algorítmicos a velocidades inalcanzables por la mente humana.

Resumiendo, un modelo computacional es un modelo formal (que por lo tanto puede expresarse en lenguaje matemático como un conjunto de ecuaciones), y la simulación computacional es una herramienta que nos permite estudiarlo más allá de los límites actuales de las matemáticas. De este modo, el resultado fi- 
nal es un modelo potencialmente más realista, y que todavía conserva el rigor formal de los modelos matemáticos más tradicionales.

\section{Esquema de modelado computacional de sistemas complejos}

La posibilidad de trabajar con modelos formales más complejos que los modelos matemáticos tradicionales nos obliga a expandir ligeramente el marco del proceso de modelado científico que introdujimos en la sección anterior (ver fig. 2).

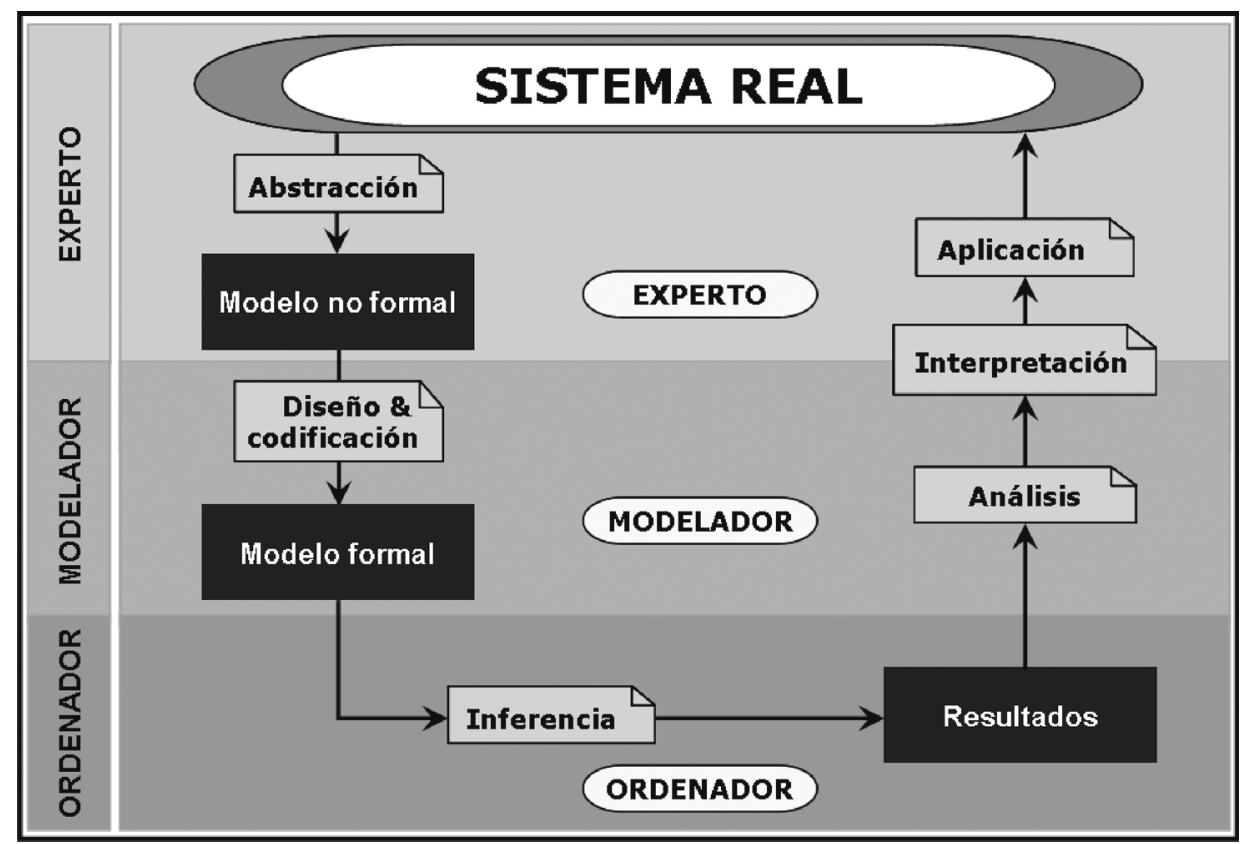

Fig. 2. Proceso de modelado con abstracción intermedia. La figura muestra un esquema secuencial por claridad pero, como se explica en la segunda sección, el proceso de modelado contiene en general varios bucles de retroalimentación.

Cuando tratamos de modelizar sistemas complejos, raramente construimos un modelo formal directamente sobre el sistema real. Normalmente la mayoría de los científicos abstraemos, consciente o inconscientemente, un modelo intermedio de cómo creemos que el sistema real funciona, y es esta abstracción - a menudo incompleta y no formal - la que modelizamos formalmente (fig. 2). Lamentablemente, no es frecuente encontrar artículos científicos en los que este primer modelo no formal se hace explícito. De hecho, esta primera abstracción suele permanecer eternamente en el etéreo ámbito de los modelos mentales. 
Cuando existen una o más capas intermedias entre el sistema real y nuestro modelo formal, la secuencia básica de modelado se ve modificada sustancialmente (Edmonds et al., 2001; Galán et al., 2008). Independientemente de la naturaleza o complejidad del sistema real, nuestro objetivo sigue siendo el mismo: avanzar nuestro conocimiento sobre el funcionamiento del sistema real mediante la construcción y el análisis de modelos. Para explicar cada una de las etapas del proceso de modelado de sistemas complejos encontramos útil distinguir tres roles (Drogoul et al., 2003): experto, modelador y ordenador (ver fig. 2). Es importante resaltar que a menudo es una única persona la que asume los roles de experto y de modelador (y hasta a veces incluso el rol del ordenador), pero no siempre es así.

El experto es una persona con un gran conocimiento del sistema real que se pretende modelizar y de su funcionamiento. Sin embargo, el experto no está necesariamente familiarizado con la creación y el uso de modelos formales. El modelador, por el contrario, es un profesional cuya labor principal consiste en diseñar, implementar y analizar modelos formales. El modelador tiene, por tanto, conocimientos avanzados de algún formalismo que permita crear y estudiar modelos formales (p. ej. cálculo simbólico, lógica proposicional, o un lenguaje de programación). Finalmente, el ordenador es el encargado de ejecutar o resolver el modelo formal, i.e. el ordenador deduce las implicaciones lógicas que se derivan de las premisas que definen el modelo y de las condiciones iniciales. Tradicionalmente, esta labor deductiva venía siendo realizada por matemáticos, quienes a menudo eran capaces de resolver el modelo formal para casos muy generales. En el caso de modelos de sistemas complejos — cuya resolución matemática general es en muchas ocasiones inviable- es un ordenador el que normalmente asume este rol. Una vez presentados los tres roles, pasamos a explicar brevemente cada una de las etapas del proceso de modelado (fig. 2).

Abstracción. La primera etapa es el proceso de abstracción, el cual nos conduce a un primer modelo que recoge los aspectos más relevantes del sistema real. Esta primera actividad es realizada por el experto, comienza con la observación del sistema real (que a menudo incluye una extracción de datos), y finaliza con la primera conceptualización del sistema objetivo. El trabajo del experto consiste en definir los objetivos concretos que se pretenden alcanzar con el proceso de modelado, identificar los componentes más importantes del sistema y las interacciones que pueda existir entre ellos, y describir las relaciones causales más significativas.

El resultado de la labor del experto es un modelo normalmente no formal, frecuentemente expresado en lenguaje natural, y que a menudo incluye diagramas conceptuales sencillos (p. ej. diagramas de bloques). Es posible que esta primera abstracción no sea completa, i.e. use términos ambiguos como «aprendizaje»o «imitación» sin especificar de manera precisa cómo se producen exactamente. Además, puesto que a menudo este primer modelo está expresado en lenguaje natural, es posible que ni siquiera tenga coherencia lógica interna. Lo 
que sí que debe recoger de manera clara e inequívoca esta primera abstracción es el conjunto de componentes, interacciones y variables críticas del sistema.

Diseño \& Codificación. La segunda etapa consiste en diseñar e implementar un modelo formal a partir de la abstracción llevada a cabo por el experto. Esta tarea corre a cargo de la persona que asume el rol del modelador (que potencialmente es la misma que asumió el rol de experto), y supone dos retos fundamentales que explicamos a continuación.

El primer reto aparece cuando el modelo creado por el experto no está completamente especificado, algo que ocurre con sorprendente frecuencia. Imagine, por ejemplo, que el experto explica que una población evoluciona de forma que los individuos con más recursos tienden a reproducirse más que aquellos con menos recursos. Obviamente, existe una infinidad de algoritmos diferentes que implementan esta idea: la relación entre recursos y número de descendientes puede ser lineal, cuadrática, exponencial, etc. Todas estas relaciones satisfacen la premisa del experto $-\mathrm{y}$ por lo tanto son en principio igualmente válidaspero asumir una u otra conducirá en general a diferentes resultados al ejecutar el modelo formal. En otras palabras, a menudo existen multitud de modelos formales diferentes que se ajustan perfectamente a las especificaciones del experto.

El segundo reto surge cuando el modelo proporcionado por el experto carece de coherencia interna, algo que puede ocurrir perfectamente puesto que es frecuente que la abstracción del experto esté expresada en lenguaje natural. Descubrir incoherencias lógicas en modelos no formales no es una tarea trivial. Varios autores (p. ej. Christley et al. (2004), Pignotti et al. (2005), y Polhill et al. (2007)) han enfatizado la utilidad de ontologías para llevar a cabo esta tarea, particularmente en el dominio de las simulaciones sociales computacionales. En cualquier caso, estos dos retos para el modelador ponen de manifiesto el enorme valor que tiene formalizar modelos.

En resumen, el modelador deberá tratar de diseñar e implementar un modelo o conjunto de modelos formales tales que (a) cada uno de ellos sea una particularización válida de las especificaciones del experto y (b) todos ellos en conjunto constituyan una expresión representativa de la abstracción del experto. Para ello, el modelador, a menudo en constante comunicación con el experto, deberá acordar y realizar varias suposiciones de naturaleza muy diversa. Algunas suposiciones vendrán impuestas por la necesidad de producir un modelo completamente especificado, y darán lugar a particularizaciones concretas de ideas generales expresadas por el experto. Otras suposiciones serán necesarias para poder ejecutar el modelo con la tecnología disponible (p. ej. representar un espacio tridimensional como un conjunto de celdas bidimensionales). Finalmente, es posible que haya suposiciones implícitamente codificadas en el modelo formal y que ni siquiera el propio modelador sea plenamente consciente de que las está imponiendo (p. ej. el uso de aritmética de punto flotante en vez de aritmética real).

Una vez diseñado el modelo formal, el modelador podrá implementarlo en multitud de formalismos diferentes. En particular, todo modelo computacional 
puede expresarse como un conjunto de ecuaciones matemáticas (Leombruni \& Richiardi, 2005; Epstein, 2006) y también puede re-implementarse en cualquier lenguaje de programación que permita el uso de secuenciación, selección e iteración (Böhm \& Jacopini, 1966; Harel, 1980). Diferentes re-implementaciones son simplemente diferentes formas de representar la misma relación entrada-salida.

Conviene mencionar que cuando el experto y el modelador son roles ocupados por la misma persona (algo que ocurre frecuentemente), el modelo no formal no suele ser explícito (y mucho menos estar publicado). No obstante, en ocasiones son dos personas diferentes las que asumen sendos roles, y entonces resulta sencillo distinguir el modelo no formal propuesto por el experto del modelo formal desarrollado por el modelador. El desarrollo de la teoría clásica de difusión de innovaciones desarrollada en los años sesenta representa un interesante ejemplo. En 1962 Everett M. Rogers publicó su conocido libro Diffusion of Innovation (Rogers, 1962), en el que propone un completo y descriptivo marco conceptual sobre la difusión de innovaciones. En este marco conceptual, que es fundamentalmente discursivo y no formal, Rogers clasifica los elementos fundamentales del proceso de difusión en cuatro categorías: la naturaleza de la propia innovación, el canal de comunicación, el sistema social al que se comunica, y el tiempo. Siete años más tarde, Bass (1969) publicó un influyente artículo formalizando los aspectos temporales del modelo propuesto por Rogers. El propio Bass (1969) escribió: «.. .la teoría de la adopción y difusión de nuevas ideas y productos por un sistema social ha sido discutida extensamente por Rogers (1962). Esta discusión es fundamentalmente literaria. No es, por tanto, fácil separar las premisas de la teoría de las conclusiones...». En su artículo, Bass (1969) toma el rol del modelador y construye un modelo formal a partir de la «discusión fundamentalmente literaria» (o modelo no formal) de Rogers (1962).

Inferencia. Una vez construido el modelo formal, el siguiente paso es ejecutarlo o resolverlo. Si el modelo formal es matemáticamente resoluble, a menudo es una persona la que asume este rol de deducir las implicaciones lógicas que suponen los axiomas y las reglas que definen el modelo. Si, por el contrario, el modelo es suficientemente complejo, puede ser aconsejable explorar las implicaciones lógicas mediante el uso de la simulación computacional.

Conviene enfatizar que, en cualquier caso, este proceso inferencial consiste «simplemente» en aplicar procesos deductivos con el objetivo de averiguar el conjunto de proposiciones que se derivan con necesidad lógica de los axiomas y las reglas que definen el modelo. Estas implicaciones o resultados deberían ser idénticos independientemente del formalismo usado para implementar el modelo formal; si no lo fueran, habríamos probado que los dos (o más) programas usados no están en realidad implementado el mismo modelo formal (i.e. la misma relación entrada-salida). 
Análisis. Una vez ejecutada la simulación, obtenemos unos resultados que deberemos analizar para mejorar nuestro entendimiento de cómo funciona el modelo formal que hemos creado. En general, el análisis de la dinámica de modelos de sistemas complejos no es trivial. A menudo es conveniente usar varios formalismos diferentes para estudiar el mismo modelo formal y sus resultados desde diferentes ángulos. Algunas técnicas que suelen utilizarse son: contrates de hipótesis y otras técnicas estadísticas (ver p. ej. Polhill et al., 2001; Gotts et al., 2003), teoría de procesos estocásticos (Izquierdo et al., 2008a), aproximaciones de campo medio (ver p. ej. Galán \& Izquierdo, 2005; Izquierdo et al., 2007), visualizaciones de datos interactivas (ver p. ej. Izquierdo et al., 2008a; Izquierdo et al., 2008b), o simple inspección.

Interpretación. Después de analizar los resultados obtenidos con el modelo, el modelador y/o el experto los interpretarán usando ya conceptos referentes al sistema real. Nótese que, desde el punto de vista del ordenador, los resultados obtenidos con el modelo formal carecen de significado intrínseco: son simplemente cadenas de bits deducidas a partir de otras cadenas de bits. La interpretación consiste en asignar significado a los resultados obtenidos con el modelo formal, previamente analizados.

Aplicación. Finalmente, el objetivo de todo este ejercicio de modelado no es otro que aplicar sobre el sistema real el conocimiento que hemos adquirido sobre nuestra abstracción. Como se mencionó en la sección anterior, el término aplicación no debe entenderse necesariamente en su sentido práctico más estricto. La aplicación del conocimiento adquirido puede ser tan concreta como una predicción numérica o tan vaga como una indicación de algún posible comportamiento en términos cualitativos. El lector puede encontrar varios ejemplos en p. ej. Shan \& Yang (2008).

Conviene resaltar que todo conocimiento que podamos extraer de manera formal y libre de error se referirá necesariamente al modelo formal, no a la primera abstracción ni, por supuesto, al sistema real. Una imprudencia que debemos evitar en el modelado de sistemas complejos consiste en combinar mentalmente nuestra primera abstracción no formal y el sistema real, e intentar interpretar los resultados de nuestro modelo formal directamente sobre el sistema real. Este tipo de tentaciones nos puede conducir a extrapolar nuestras conclusiones sobre el modelo formal a ámbitos donde dichas conclusiones pueden no ser válidas (ver p. ej. Edmonds \& Hales, 2005; Galán \& Izquierdo, 2005).

Las dos secciones siguientes recogen las principales características de dos técnicas de modelado computacional: la simulación basada en agentes y la dinámica de sistemas. Posteriormente, estas dos técnicas son comparadas bajo el marco general del proceso de modelado científico descrito en esta sección. 


\section{SIMULACIÓN BASADA EN AGENTES}

La simulación basada en agentes ha demostrado ser una técnica tremendamente útil para modelizar sistemas complejos, y muy especialmente sistemas sociales (Conte et al., 1997; Gilbert \& Troitzsch, 1999; Gilbert \& Terna, 2000; Gilbert, 2007). Mediante la simulación basada en agentes, el modelador reconoce explícitamente que los sistemas complejos, y en particular los sociales, son producto de comportamientos individuales y de sus interacciones.

Lo que distingue a la simulación basada en agentes de otras técnicas de modelado es la forma en que se construye la primera abstracción del sistema real $\mathrm{y}$, consecuentemente, el modelo formal (ver fig. 2). En los modelos formales construidos mediante simulación basada en agentes, los componentes básicos del sistema real están explícita e individualmente representados en el modelo (Edmonds et al., 2001). De esta forma, como se muestra en la figura 3, las fronteras

\section{Sistema objetivo \\ Modelo basado en agentes}
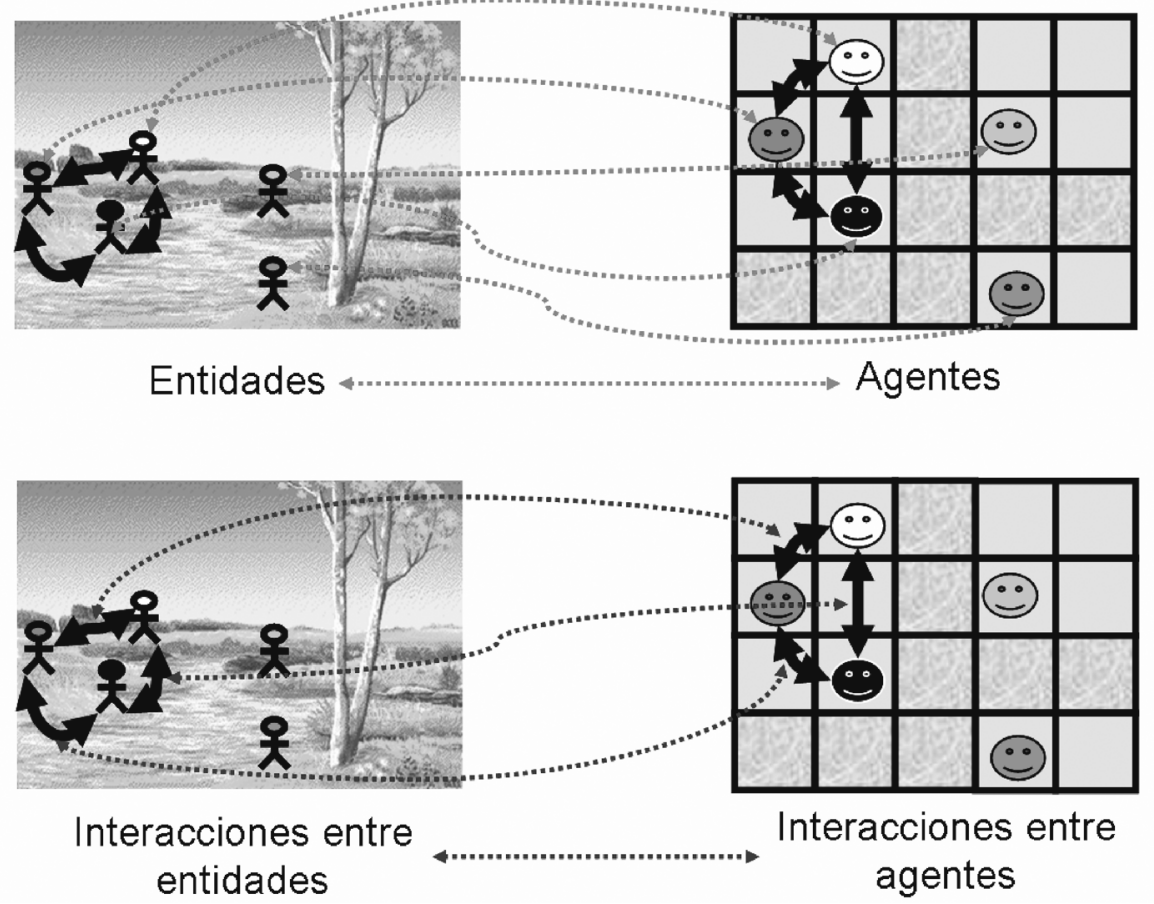

Fig. 3. En los modelos formales construidos mediante simulación basada en agentes, los componentes básicos del sistema real y las interacciones entre ellos están explícita e individualmente representados en el modelo. 
que definen a los componentes básicos del sistema real se corresponden con las fronteras que definen a los agentes del modelo, y las interacciones que tienen lugar entre los componentes básicos del sistema real se corresponden con las interacciones que tienen lugar entre los agentes del modelo (Edmonds et al., 2001; Galán et al., 2008). Esta correspondencia directa contrasta con el tradicional uso de 'agentes representativos' y es capaz de aumentar el realismo y el rigor científico de los modelos formales así construidos.

Los sistemas basados en agentes se caracterizan por comprender varios agentes que son - en mayor o menor grado- autónomos, heterogéneos e independientes, que muestran cada uno sus propias metas y objetivos, y que generalmente son capaces de interaccionar entre sí y con su entorno (Torsun, 1995). En muchas ocasiones, pero no siempre, son sistemas caracterizados por la existencia de un número grande de agentes relativamente simples, que pueden evolucionar a lo largo del tiempo para adaptarse a nuevas condiciones del entorno o a nuevos objetivos. En particular, la simulación basada en agentes es especialmente relevante en sistemas complejos con las siguientes características:

- Sistemas con componentes individuales heterogéneos; especialmente aquéllos en los que las implicaciones de esta heterogeneidad no se han estudiado en profundidad (Axtell, 2000). Varias disciplinas (p. ej. la economía neoclásica) utilizan frecuentemente la hipótesis de 'individuo prototipo' o 'agente representativo', confiando en que los resultados obtenidos bajo esta hipótesis puedan aplicarse satisfactoriamente a sistemas reales en los que los individuos son, en realidad, heterogéneos. Sin embargo, en sistemas caracterizados por fuertes externalidades (p. ej. la explotación del medio ambiente, la gestión de recursos comunes...), los resultados obtenidos usando modelos que asumen la existencia de un individuo representativo están, en muchas ocasiones, muy alejados de las observaciones empíricas (ver p ej. Ostrom et al., 1994). Ignorar por principio la existencia de heterogeneidad entre individuos es una fuerte premisa que debe ser contrastada; para ello, resulta necesario estudiar el sistema con heterogeneidad, y el modelado basado en agentes es particularmente útil para acometer este análisis.

- Sistemas adaptativos, i.e. sistemas en los que los componentes individuales del sistema son capaces de aprender (adaptación a escala individual), o bien pueden ser seleccionados y reemplazados de acuerdo con algún criterio (adaptación a nivel poblacional). En cualquiera de estos dos casos parece claro que resulta conveniente representar explícita e individualmente cada componente del sistema.

- Sistemas en los que el espacio geográfico puede tener una influencia significativa. En muchos sistemas, el hecho de que dos individuos estén separados en el espacio supone una probabilidad de interacción inferior. El modelado basado en agentes facilita la representación del espacio físico en el que se mueven e interactúan los agentes. 
- Sistemas en los que existen redes sociales de interacción. Las interacciones entre componentes del sistema pueden estar influenciadas por diversos factores además de por el espacio físico. El modelado basado en agentes facilita la representación explícita de redes de interacción social que no están necesariamente estructuradas espacialmente.

- Sistemas en los que se desea analizar en profundidad la relación existente entre los atributos y comportamientos de los individuos (la 'micro-escala') frente a las propiedades globales del grupo (la 'macro-escala') (Gilbert \& Troitzsch, 1999; Squazzoni, 2008).

La metodología basada en agentes ha sido utilizada ampliamente para modelizar sistemas en un amplio rango de disciplinas científicas: por ejemplo, economía (Tesfatsion, 2002, 2003), finanzas (LeBaron, 2000), gestión de recursos naturales y ecología (Bousquet \& Le Page, 2004; López \& Hernández, 2008), ciencias políticas (Axelrod, 1997b; Johnson, 1999), antropología (Kohler \& Gumerman, 2000), sociología (Conte et al., 1997; Gilbert \& Troitzsch, 1999; Gilbert, 2007), biología (Paton et al., 2004; Walker et al., 2004a; Walker et al., 2004b) o medicina (Mansury et al., 2002; Mansury \& Deisboeck, 2004), en los que partiendo de reglas que determinan el comportamiento individual de los agentes se pretende inferir las propiedades globales de todo el sistema (Holland, 1998). Los métodos basados en agentes facilitan el estudio y modelado de sistemas complejos a partir de las unidades que los componen, permitiéndonos construir modelos experimentales de la realidad desde un punto de vista diferente al tradicional: desde lo más simple hacia lo más complejo.

Sin duda, uno de los puntos fundamentales de la simulación basada en agentes es el concepto de emergencia. Los fenómenos emergentes son patrones macroscópicos que surgen a partir de las interacciones descentralizadas de componentes individuales más simples (Holland, 1998). Lo que caracteriza a estos fenómenos emergentes es que su presencia o aparición no resulta evidente a partir de una descripción del sistema consistente en la especificación del comportamiento de sus componentes individuales y de las reglas de interacción entre ellos (Gilbert \& Terna, 2000; Gilbert, 2002; Squazzoni, 2008). Un ejemplo típico de fenómeno emergente es la formación de grupos diferenciados en el modelo de segregación de Schelling (1971); la aparición de patrones claros de segregación no está explícitamente impuesta en la definición del modelo, sino que emerge de las interacciones locales de individuos con tendencias segregacionistas en ocasiones sorprendentemente débiles. Otro ejemplo son los patrones migratorios de Sugarscape (Epstein \& Axtell, 1996).

Existen multitud de fenómenos emergentes en diversas disciplinas (ver p. ej. Reynolds, 1987; Holland, 1998; Johnson, 2001), pero sin duda es en las ciencias sociales donde la idea de emergencia cobra una dimensión adicional de complejidad e importancia. En muchos sistemas sociales en los que interviene la dimensión humana cabe la posibilidad de que cada uno de los componentes individuales del sistema tome cierta consciencia del fenómeno emergente del que es 
causa parcial y que, como consecuencia de esta percepción consciente, reaccione modificando su comportamiento. Este fenómeno - conocido como emergencia de segundo orden (Gilbert, 1995; Gilbert, 2002; Squazzoni, 2008)— subyace tras la complejidad de muchos sistemas sociales.

Los mercados financieros constituyen un ejemplo claro de emergencia de segundo orden. En estos mercados, el precio (fenómeno emergente) surge de la interacción de varios inversores (componentes individuales del sistema); los inversores perciben de forma consciente el precio que ellos mismos generan, y modifican su comportamiento en respuesta a esta observación, creando así un complejo lazo de realimentación entre los dos niveles jerárquicos del sistema.

Puesto que el énfasis en la simulación basada en agentes está en encontrar abstracciones apropiadas que describan los componentes básicos del sistema y sus interacciones (en vez de buscar abstracciones que versen directamente sobre la dinámica global del sistema), esta técnica de modelado es particularmente útil para modelar procesos emergentes de forma natural.

\section{DINÁMICA DE SISTEMAS}

La dinámica de sistemas es otra técnica de modelado de sistemas complejos. También se utiliza ampliamente para modelizar sistemas en ingeniería (Ford, 1997; Ford \& Sterman, 1998), economía y negocios (Sterman, 2000; Ellis, 2007), planificación y gestión de proyectos (Rodrigues \& Bowers, 1996; Lyneis \& Ford, 2007), gestión medioambiental (Martínez \& Esteve, 2007), sanidad pública (Homer \& Hirsch, 2006) e incluso historia (Cruz, 2007), por poner algunos ejemplos.

A diferencia de la simulación basada en agentes, la filosofía de la dinámica de sistemas gira en torno al concepto de retroalimentación, o causalidad circular entre variables observables. Estas variables observables pueden describir algún atributo de los componentes básicos del sistema (p. ej. la velocidad de una partícula de gas en un recipiente), o referirse a alguna magnitud global del sistema (la presión en el recipiente). Bien es cierto que existe una tendencia generalizada en la dinámica de sistemas a utilizar variables observables del sistema en su conjunto. En ese caso, es importante darse cuenta desde un principio que el mero hecho de estudiar magnitudes agregadas del sistema global (en vez de estudiar magnitudes de sus componentes básicos directamente) supone ya un alto grado de abstracción.

A diferencia de la simulación basada en agentes (donde se estudian interacciones entre los componentes básicos del sistema), el foco de atención en la dinámica de sistemas reside en la relación existente entre variables observables. Estas relaciones son, en general, suficientemente complejas de por sí. En la mayoría de los sistemas complejos existen variables observables que no están ligadas por una relación lineal causa-efecto. Las relaciones lineales causa-efecto en el sentido tradicional del término se caracterizan por las siguientes propiedades (Scholl, 2001): 
1. la causa precede al efecto en el tiempo,

2. existe una fuerte correlación empírica entre causa y efecto, y

3. esta relación no es el resultado de ninguna otra variable.

Los sistemas complejos, lejos de caracterizarse por mostrar relaciones lineales de causalidad, suelen presentar causas y efectos que no se encuentran próximos en el espacio ni en el tiempo, y que se retroalimentan en el sistema, haciendo su análisis mediante enfoques tradicionales extremadamente complejo. Tampoco es extraño en estos sistemas observar dos variables que, pese a no mantener relación alguna de causalidad, presentan alta correlación temporal simplemente como consecuencia de la dinámica global del sistema. La dinámica de sistemas es una técnica particularmente útil para analizar este tipo de sistemas caracterizados por contener complejas relaciones de causalidad circular.

La dinámica de sistemas estudia variables observables de sistemas complejos e intenta identificar las relaciones de causalidad (normalmente circulares) que existen entre ellas. Una vez que estas relaciones están identificadas, resulta más sencillo explicar el origen de comportamientos globales del sistema a partir de su estructura causal. Del mismo modo, también resulta más sencillo identificar las acciones que deben tomarse para modificar artificialmente la evolución de alguna de las variables modeladas. En muchas ocasiones, esta intervención externa consiste en modificar la estructura de causalidad existente entre las variables del sistema.

Debido a la estructura de los modelos formales creados con un enfoque de dinámica de sistemas, a menudo resulta especialmente sencillo representarlos usando lenguaje matemático tradicional. En otras palabras, la mayoría de los modelos de dinámica de sistemas pueden expresarse sin gran esfuerzo como un conjunto de ecuaciones algebraicas (a menudo diferenciales) cuyas variables son propiedades (en general macroscópicas) del sistema modelado.

El énfasis en la dinámica de sistemas está, por tanto, en encontrar las variables críticas del sistema complejo e identificar los vínculos causales que existen entre ellas. El proceso de abstracción que nos lleva de los componentes básicos del sistema y sus interacciones hasta las variables críticas del mismo corre, en este caso, a cargo del experto o del modelador, y es previo a la creación del modelo formal. Los componentes básicos del sistema global no suelen estar explícita e individualmente representados en el modelo formal.

\section{DIFERENCIAS ENTRE SIMULACIÓN BASADA EN AGENTES Y DINÁMICA DE SISTEMAS}

La primera y más fundamental diferencia que puede surgir entre dos técnicas de modelado aparece en el proceso de abstracción (ver fig. 2). Si éste es diferente, la abstracción del sistema real obtenida será diferente y, por lo tanto, todas las etapas que siguen en el proceso de modelado científico (diseño, codificación, 
inferencia, análisis, interpretación y aplicación) se verán alteradas. La diferencia en el proceso de abstracción es particularmente evidente entre la simulación basada en agentes y la dinámica de sistemas.

Conviene destacar aquí que es perfectamente posible tener un único modelo en el que las dos filosofías de modelado se encuentran presentes (en distintas partes o módulos del modelo). De hecho, es relativamente común desarrollar modelos en los que un grupo de agentes - representados individual y explícitamente- interaccionan en un entorno en el que ciertas variables (p. ej. aquellas que se refieren a fenómenos naturales) evolucionan siguiendo un enfoque de dinámica de sistemas. Por ello, la discusión que sigue no debe entenderse necesariamente para un modelo en su totalidad, sino potencialmente para cada uno de sus módulos, ya que éstos pueden haberse implementado siguiendo paradigmas diferentes.

Como hemos visto en las secciones anteriores, la simulación basada en agentes se centra en los componentes básicos del sistema. El proceso de abstracción se hace sobre cada componente básico individualmente, no sobre el sistema en su conjunto. Para construir un modelo basado en agentes debemos identificar los componentes básicos del sistema real, y abstraer sus propiedades individuales y las interacciones que se producen entre ellos. Estas interacciones se producen bien directamente entre los componentes básicos del sistema o a través de un entorno compartido (mediante competencia por recursos, por ejemplo). Podemos decir entonces que ni el experto ni el modelador imponen condiciones sobre el comportamiento global del sistema directamente, sino que éste emerge como consecuencia de las condiciones impuestas sobre los componentes básicos del sistema y sus interacciones. El comportamiento global del sistema no es abstraído, sino que emerge durante el proceso de inferencia, al ejecutar el modelo.

El enfoque de la dinámica de sistemas, por el contrario, se centra en las relaciones causales que ligan variables observables. Estas relaciones pueden expresarse fácilmente con ecuaciones algebraicas y es la particularización de valores que satisfacen estas ecuaciones lo que genera la dinámica global del sistema. Las ecuaciones pueden tener en cuenta variabilidad en el tiempo (ecuaciones diferenciales) y en el espacio (ecuaciones en diferencias parciales). El modelador que usa dinámica de sistemas puede ser perfectamente consciente de que las relaciones entre las variables observables son fruto de las interacciones no lineales entre los componentes básicos del sistema, pero no lo reproduce explícitamente en su modelo; el modelador (y quizá previamente el experto) se abstrae de estas interacciones. Los intentos formales de traducción entre modelos basados en agentes y modelos de dinámica de sistemas sólo surgen de forma general y natural cuando es posible discretizar los stocks de la dinámica de sistemas en actores individuales del sistema (Borshchev et al., 2004).

De un modo un tanto informal, podemos decir que la simulación basada en agentes simula actores y los deja interactuar para crear la película, mientras que la dinámica de sistemas simula el guión de la película directamente. 
Otra diferencia fundamental que existe entre la simulación basada en agentes y la dinámica de sistemas radica en el grado de refinamiento en la definición de componentes básicos y de variables observables. Si bien esta diferencia no tiene por qué darse en teoría, lo cierto es que existe en la práctica. La mayoría de los modelos de dinámica de sistemas se centran en variables observables del sistema agregado (Parunak et al., 1998). En simulación basada en agentes, por el contrario, el énfasis radica en definir el comportamiento de los agentes, que no está necesariamente determinado por variables agregadas del sistema, sino que puede basarse sólo en información local. En simulación basada en agentes las variables observables del sistema agregado surgen como resultado de la interacción de los agentes. Es posible que los agentes puedan ser capaces de observar las variables agregadas y modificar su comportamiento en consecuencia (i.e. emergencia de segundo orden), pero lo importante es darse cuenta de que en simulación basada en agentes el proceso de emergencia que lleva de los componentes individuales a las variables agregadas se modela explícitamente.

Como es de esperar, estas diferencias en el proceso de abstracción y de diseño del modelo repercuten directamente en el resto de las etapas del proceso de modelado científico. A la hora de codificar el modelo computacional, si bien es cierto que éste puede expresarse en multitud de formalismos diferentes (en particular, en cualquier lenguaje de programación suficientemente sofisticado), también es cierto que la implementación en determinados formalismos puede ser mucho más natural que en otros. Por ejemplo, los lenguajes de programación orientados a objetos (p. ej. Java, C++, Objective-C ó SDML (Moss et al., 1998)) son particularmente útiles para construir modelos basados en agentes.

También existen formalismos y bibliotecas de código que han sido creadas específicamente para facilitar la creación de modelos de acuerdo con un paradigma u otro. Por ejemplo, para simulación basada en agentes son particularmente útiles las plataformas de desarrollo NetLogo (Wilensky, 1999) y Ascape (Parker, 2001), las bibliotecas de código Swarm (Nelson et al., 1996), Repast (North et al., 2006) y Mason (Luke et al., 2005), o las recientes adaptaciones de plataformas y metodologías de sistemas multi-agente hacia la simulación social (Sansores \& Pavón, 2005; Sansores et al., 2006; Arroyo \& Hassan, 2007). Railsback et al. (2006) comparan Netlogo, Swarm, Repast y Mason. El proceso de diseño y codificación de un modelo de dinámica de sistemas es normalmente más sencillo, en parte porque los modelos formales suelen ser menos complejos, y en parte gracias a la disponibilidad de herramientas informáticas de muy alto nivel para construir y analizar este tipo de modelos (p. ej. Vensim (2008) y NetLogo (Wilensky, 1999)). Según algunos autores (Parunak et al., 1998), esta facilidad de construcción y análisis de modelos de dinámica de sistemas mediante herramientas 'drag-and-drop' ha sido una de las principales razones de su enorme popularidad en la comunidad científica.

La etapa de inferencia suele ser llevada a cabo por el ordenador. Las posibles diferencias que puedan surgir en esta etapa entre las dos metodologías serán debidas a la plataforma escogida para implementar, ejecutar y analizar el modelo 
formal. NetLogo (Wilensky, 1999) es una plataforma que nos ofrece algo particularmente interesante para nuestros propósitos: comparar en tiempo real de ejecución, y dentro de la misma plataforma, un modelo diseñado con un enfoque basado en agentes y otro diseñado bajo la óptica de dinámica de sistemas.

Posteriormente se analizan los resultados obtenidos, normalmente haciendo uso de técnicas estadísticas y otras herramientas informáticas más sofisticadas. El resto de las etapas del proceso de modelado científico (interpretación y aplicación) son demasiado específicas para cada sistema complejo como para tratar de compararlas en un ensayo general sobre metodologías.

\section{CONCLUSIONES}

En esta última sección explicamos en detalle el principal factor que - a nuestro parecer- determina cuál de las dos técnicas estudiadas en este artículo es probable que resulte más útil a la hora de modelar un determinado sistema complejo. Hemos mostrado que la principal diferencia entre la simulación basada en agentes y la dinámica de sistemas radica en la forma en que se construye la primera abstracción del sistema real objeto de estudio. Por tanto, resulta claro que el principal factor que determina cuál de las dos técnicas será más útil viene determinado por lo apropiada que sea esta primera abstracción dados los objetivos del proceso de modelado. Sin duda, un segundo factor que también afecta la elección en la práctica es la disponibilidad de herramientas para construir un modelo de acuerdo con un paradigma u otro (y, sobre todo, la pericia del modelador en el uso de estas herramientas), pero entendemos que este factor no precisa de más explicación.

Como hemos visto, la simulación basada en agentes realiza abstracciones directamente sobre los componentes básicos del sistema. La dinámica de sistemas, por el contrario, versa sobre variables (normalmente agregadas) del sistema y sobre las relaciones causales que las ligan. Esta diferencia tiene una implicación fundamental en lo que se refiere al proceso de emergencia que nos lleva desde las interacciones locales entre los componentes básicos del sistema hasta las relaciones globales entre las variables agregadas del mismo. En simulación basada en agentes este proceso de emergencia se modela explícitamente, mientras que en dinámica de sistemas se presupone a priori (y son las relaciones entre las variables agregadas las que se modelan explícitamente a una escala superior).

Si tanto (a) nuestro conocimiento previo del sistema complejo, como (b) los objetivos que pretendemos alcanzar con el proceso de modelado, nos permiten llevar cabo la abstracción de este proceso de emergencia de forma sólida y fundada, entonces la dinámica de sistemas será normalmente más conveniente. En general, la dinámica de sistemas —al ofrecer un grado de abstracción superior al desarrollado en los modelos basados en agentes - dará lugar a modelos de complejidad inferior, lo que facilitará su implementación, su análisis y su interpretación. Sin embargo, si la abstracción del proceso de emergencia no puede 
llevarse a cabo a priori de forma científicamente válida dados nuestros objetivos, entonces será más apropiado modelar el proceso de emergencia explícitamente (mediante la simulación basada en agentes) para, de esta forma, poder estudiarlo en detalle. El modelo así construido será científicamente más riguroso pero notablemente más complejo, con los inconvenientes que esto conlleva.

En muchas ocasiones es el propio proceso de emergencia el que se pretende estudiar en detalle, con lo cual el enfoque basado en agentes es nuestra única opción. En particular, si se quiere contrastar la validez de la abstracción del proceso de emergencia que se lleva a cabo con dinámica de sistemas, entonces un enfoque basado en agentes es la única alternativa.

Conviene enfatizar que los objetivos que se pretenden alcanzar con el proceso de modelado juegan un papel fundamental a la hora de considerar qué técnica de modelado puede resultar más útil. El siguiente ejemplo es revelador: considere una red social de $N$ individuos en forma de anillo (o mesa redonda), donde cada individuo es amigo de los dos individuos que tiene a cada uno de sus lados. Al comienzo de la simulación $(t=0)$ sólo uno de los individuos conoce una cierta información. La única regla del modelo es que si un individuo conoce la información en un instante $t=t_{0}$, entonces sus dos amigos también la conocen en el instante siguiente $t=t_{0}+1$. Si nuestro único objetivo como modeladores es conocer el número de individuos $n(t)$ que conocen la información en cada instante arbitrario $t$, entonces un enfoque de dinámica de sistemas será suficiente, puesto que sabemos que $n(0)=1$, y además:

$$
\begin{array}{ll}
n(t+1)=n(t)+2 & \text { si } n(t)<N-1 \\
n(t+1)=N & \text { si } n(t) \geq N-1
\end{array}
$$

Sin embargo, si nuestro objetivo incluye además poder identificar exactamente qué individuos conocen la noticia en un instante $t$ (sabiendo quién la conocía en algún instante previo), entonces será necesario modelar la red social de individuos explícitamente, lo que supone un enfoque basado en agentes. El sistema real que se modela es exactamente el mismo, y es sólo el objetivo del ejercicio de modelado el que determina qué técnica resulta más útil.

Naturalmente, hay sistemas en los que los procesos de emergencia son notablemente más simples que en otros. Esto hace que —en términos muy generales - podamos dar una idea sobre las características particulares de un sistema que - en principio - pueden sugerir cuál de las dos técnicas de modelado es probable que resulte más útil (teniendo en cuenta los objetivos que se pretenden alcanzar con el modelo). A grandes rasgos, podemos decir que generalmente la dinámica de sistemas resulta más apropiada para modelizar sistemas compuestos por entidades homogéneas, dominados por leyes generales uniformes en el tiempo y en el espacio (como las leyes físicas), y que pueden modelizarse correctamente de una forma centralizada. En sistemas más complejos, caracterizados por un alto grado de localización y heterogeneidad de sus componentes individuales, y dominados por procesos de intercambio de información locales, 
asimétricos y descentralizados (como la mayor parte de los sistemas sociales), conviene contemplar la posibilidad de desarrollar un enfoque basado en agentes. El paradigma de modelado utilizado también se verá influenciado por el tipo de información disponible sobre el sistema, puesto que dicha información determinará la validación empírica de los modelos formales obtenidos.

En cualquier caso, la validez científica de las abstracciones que realizamos sobre el mundo real es siempre discutible, por lo que, en la mayoría de las ocasiones, el procedimiento idóneo es simular el mismo sistema haciendo uso de las dos técnicas y comparar los resultados obtenidos. Algunos autores que ya han realizado este tipo de estudios (Parunak et al., 1998; Scholl, 2001) coinciden en recomendar el modelado de ciertos sistemas complejos haciendo uso de las dos metodologías.

\section{AGRADECIMIENTOS}

Los autores se han beneficiado enormemente de multitud de discusiones con Segismundo S. Izquierdo. Este trabajo se deriva de la participación de sus autores en los proyectos de investigación financiados por el Ministerio de Educación y Ciencia con referencias DPI2004-06590 y DPI2005-05676, titulados «Integración empresarial y gestión de la cadena de suministro basada en sistemas multiagente» $\mathrm{y}$ «Simulador basado en agentes para la gestión del agua en espacios metropolitanos».

\section{BIBLIOGRAFÍA}

Armatte, M. (2006): «La Noción de Modelo en las Ciencias Sociales». Empiria: Revista de metodología de ciencias sociales 11, pp. 33-70.

ARroyo, M. \& HASSAN, S. (2007): «Simulación de procesos sociales basada en agentes software». Empiria: Revista de metodología de ciencias sociales 14, pp. 139-161.

AXELrod, R. (1997a): Advancing the Art of Simulation in the Social Sciences. En Conte R, Hegselmann, R. \& Terna, P. (Eds.) Simulating Social Phenomena, Lecture Notes in Economics and Mathematical Systems 456: 21-40. Berlin: Springer-Verlag.

- (1997b): The complexity of cooperation: Agent based models of competition and collaboration. Princeton NJ: Princeton University Press.

AXTELL, R. (2000): «Why agents? On the varied motivations for agent computing in the social sciences». En Macal, C. M. \& Sallach, D. (eds.), Proceedings of the Workshop on Agent Simulation: Applications, Models, and Tools, pp. 3-24. Argonne, IL: Argonne National Laboratory.

BASs, F. M. (1969): «A new product growth for model consumer durables». Management Science 15(5), pp. 215-227.

Bertalanffy, L. (1968): General System Theory. New York: George Braziller Publisher.

BÖHM, C. \& JACOPINI, G. (1966): «Flow diagrams, turing machines and languages with only two formation rules». Communications of the ACM 9(5), pp. 366-371. 
Borshchev, A., Filippov, A., Kennedy, M., Winch, W. G., Langer, R. S., Rowe, J. I. \& YANNI, J. M. (2004): «From System Dynamics and Discrete Event to Practical Agent Based Modeling: Reasons, Techniques,Tools». En Proceedings of the 22nd International Conference of the System Dynamics Society. Albany, NY: Systems Dynamics Society.

Bousquet, F. \& Le Page, C. (2004): «Multi-agent simulations and ecosystem management: A review». Ecological Modelling 176(3-4), pp. 313-332.

Conte, R., Hegselmann, R. \& Terna, P. (1997): «Simulating Social Phenomena». Lecture Notes in Economics and Mathematical Systems 456.

Cruz, M. M. B. (2007): «Evaluando la dinámica de sistemas como una herramienta para enseñar Historia». Revista de Dinámica de Sistemas 3(2), pp. 3-60.

Christley, S., XiAng, X. \& MAdey, G. (2004): «Ontology for agent-based modeling and simulation». Agent 2004 Conference on Social Dynamics: Interaction, Reflexivity and Emergence, Chicago, IL. Argonne National Laboratory \& The University of Chicago.

Drogoul, A., Vanbergue, D. \& Meurisse, T. (2003): «Multi-Agent Based Simulation: Where are the Agents?» En Sichman, J. S., Bousquet, F. \& Davidsson, P. (eds.), Lecture Notes in Computer Science 2581. Proceedings of MABS 2002 Multi-AgentBased Simulation, pp. 1-15. Bologna, Italy: Springer-Verlag.

EDMONDS, B. \& HALES, D. (2005): «Computational simulation as theoretical experiment». Journal of Mathematical Sociology 29(3), pp. 209-232.

EDMONDS, B., Moss, S. \& DAVIDSson, P. (2001): «The Use of Models - making MABS actually work». In, Multi-Agent-Based Simulation, Lecture Notes in Artificial Intelligence 1979, pp. 15-32. Berlin: Springer-Verlag.

ELLIS, R. E. (2007): «El impacto del comercio con China en las economías y sociedades de América Latina». Revista de Dinámica de Sistemas 3(2), pp. 85-116.

EPSTEIN, J. M. (2006): «Remarks on the Foundations of Agent-Based Generative Social Science». En Amman, H. M., Kendrick, D. A. \& Rust, J. (eds.), Handbook of Computational Economics 2, pp. 1585-1604. North-Holland.

Epstein, J. M. \& AXTELl, R. L. (1996): Growing Artificial Societies: Social Science from the Bottom Up. The MIT Press.

FORD, A. (1997): «System dynamics and the electric power industry». System Dynamics Review 13(1), pp. 57-85.

ForD, D. N. \& STERMAN, J. D. (1998): «Dynamic modeling of product development processes». System Dynamics Review 14(1), pp. 31-68.

GALÁN, J. M. \& IzQuIERDO, L. R. (2005): «Appearances can be deceiving: Lessons learned re-implementing Axelrod's 'evolutionary approach to norms'». Journal of Artificial Societies and Social Simulation 8(3), Artículo 2.

Galán, J. M., IzQuierdo, L. R., IzQuierdo, S. S., SAntos, J. I., Olmo, R., LópezPAREDES, A. \& EDMONDS, B. (2008): «Errors and artefacts in agent-based modelling». Enviado a Journal of Artificial Societies and Social Simulation.

Gilbert, N. (2002): «Varieties of Emergence». En Macal, C. \& Sallach, D. (eds.), Social Agents: Ecology, Exchange, and Evolution. Agent 2002 Conference, pp. 4150. Chicago: University of Chicago and Argonne National Laboratory.

GiLbert, N. (2004): «Agent-based social simulation: Dealing with complexity». Centre for Research on Social Simulation, University of Surrey.

- (2007): Agent-Based Models. Quantitative Applications in the Social Sciences. London: SAGE Publications. 
Gilbert, N. \& Terna, P. (2000): «How to build and use agent-based models in social science». Mind and Society 1(1), pp. 57-72.

Gilbert, N. \& Troitzsch, K. G. (1999): Simulation for the Social Scientist. Buckingham, UK: Open University Press.

Gotts, N. M., Polhill, J. G. \& Law, A. N. R. (2003): «Aspiration levels in a land use simulation». Cybernetics \& Systems 34(8), pp. 663-683.

HAREL, D. (1980): «On folk theorems». Communications of the ACM 23(7), pp. 379-389.

Hesse, M. B. (1963): Models and Analogies in Science. London: Sheed and Ward.

Holland, J. H. (1998): Emergence. From chaos to order. Reading, MA: AddisonWesley.

Homer, J. B. \& Hirsch, G. B. (2006): «System Dynamics Modeling for Public Health: Background and Opportunities». American Journal of Public Health 96(3), pp. 452-458.

Hughes, R. G. (1997): «Models and Representation». Philosophy of Science 64(4), pp. S325-S336.

IzQuierdo, L. R., IzQuierdo, S. S., GALÁn, J. M. \& SANTOS, J. I. (2008a): «Techniques to understand computer simulations: Markov chain analysis». Enviado a Journal of Artificial Societies and Social Simulation.

IZQuierdo, L. R., IZQuiERdo, S. S., GotTS, N. M. \& Polhill, J. G. (2007): «Transient and asymptotic dynamics of reinforcement learning in games». Games and Economic Behavior 61(2), pp. 259-276.

IZQUIERDO, S. S., IZQUIERDO, L. R. \& GotTS, N. M. (2008b): «Reinforcement learning dynamics in social dilemmas». Journal of Artificial Societies and Social Simulation, 11(2), Articulo 1.

JoHnson, P. E. (1999): «Simulation modeling in political science». American Behavioral Scientist 42(10), pp. 1509-1530.

JoHnson, S. (2001): Emergence: the connected lives of ants, brains, cities, and software. New York: Scribner.

KLEIJNEN, J. P. C. (1995): «Verification and validation of simulation models». European Journal of Operational Research, 82(1), pp. 145-162.

Kleindorfer, G. B., O’Neill, L., \& Ganeshan, R. (1998): «Validation in simulation: Various positions in the philosophy of science». Management Science, 44(8), pp. 1087-1099.

Kohler, T. \& Gumerman, G. J. (2000): Dynamics in human and primate societies: Agent-based modeling of social and spatial processes. New York: Oxford University Press \& Santa Fe Institute.

LeBaron, B. (2000): «Agent Based Computational Finance: Suggested Readings and Early Research». Journal of Economic Dynamics \& Control 24(5-7), pp. 679-702.

LEOMBRUNI, R. \& RiCHIARDI, M. (2005): «Why are economists sceptical about agentbased simulations?» Physica A: Statistical Mechanics and its Applications 355(1), pp. 103-109.

López Paredes, A. \& Hernández Iglesias, C. (2008): Agent Based Modelling in Natural Resource Management. Insisoc. España. ISBN 978-84-205-4560-8.

Luke, S., Cioffi-Revilla, C., Panait, L., Sullivan, K. \& Balan, G. (2005): «MASON: A Multiagent Simulation Environment». Simulation 81(7), pp. 517-527.

LYNEIS, J. M. \& FORD, D. N. (2007): «System dynamics applied to project management: A survey, assessment, and directions for future research». System Dynamics Review 23(2-3), pp. 157-189. 
Mansury, Y. \& Deisboeck, T. S. (2004): «Simulating the time series of a selected gene expression profile in an agent-based tumor model». Physica D: Nonlinear Phenomena 196(1-2), pp. 193-204.

Mansury, Y., Kimura, M., Lobo, J. \& Deisboeck, T. S. (2002): «Emerging Patterns in Tumor Systems: Simulating the Dynamics of Multicellular Clusters with an Agentbased Spatial Agglomeration Model». Journal of Theoretical Biology 219(3), pp. 343-370.

Martínez, J. \& EsteVe, M. A. (2007): «Gestión integrada de cuencas costeras: dinámica de los nutrientes en la cuenca del Mar Menor (sudeste de España)». Revista de Dinámica de Sistemas 3(1), pp. 2-23.

Moss, S., Edmonds, B., \& WALLIS, S. (1997): «Validation and Verification of Computational Models with Multiple Cognitive Agents». Centre for Policy Modelling Report, No. 97-25.

Moss, S., Gaylard, H., Wallis, S. \& Edmonds, B. (1998): «SDML: A Multi-Agent Language For Organizational Modelling». Computational \& Mathematical Organization Theory 4(1), pp. 43-63.

Moss, S. (2008): «Alternative Approaches to the Empirical Validation of Agent-Based Models». Journal of Artificial Societies and Social Simulation, 11(1), Artículo 5.

Nelson, M., Burkhart, R., Langton, C. \& Askenazi, M. (1996): «The Swarm Simulation System: A Toolkit for Building Multi-Agent Simulations». SFI Working Paper 96-06-042.

North, M. J., Collier, N. T. \& Vos, J. R. (2006): «Experiences Creating Three Implementations of the Repast Agent Modeling Toolkit». ACM Transactions on Modeling and Computer Simulation 16(1), pp. 1-25.

Ostrom, E., Gardner, R. \& Walker, J. (1994): Rules, Games, and Common-Pool Resources. Ann Arbor: University of Michigan Press

PARKer, M. T. (2001): «What is Ascape and Why Should You Care?» Journal of Artificial Societies and Social Simulation 4(1), Artículo 5.

ParunaK, H. V. D., Savit, R. \& Riolo, R. L. (1998): "Agent-based modeling vs. equation-based modeling: A case study and users' guide». En Sichman, J. S., CONTE, R. \& GILbERT, N. (eds.), Multi-Agent Systems and Agent-Based Simulation, Lecture Notes in Artificial Intelligence 1534, pp. 10-25. Berlin, Germany: Springer-Verlag.

Paton, R., Gregory, R., Vlachos, C., Saunders, J. \& Wu, H. (2004): «Evolvable social agents for bacterial systems modeling». IEEE Transactions on Nanobioscience 3(3), pp. 208-216.

Pignotti, E., Edwards, P., Preece, A., Polhill, J. G. \& Gotts, N. M. (2005): «Semantic support for computational land-use modelling». 5th International Symposium on Cluster Computing and the Grid (CCGRID 2005), Cardiff, UK. Piscataway, NJ: IEEE Press.

Polhill, J. G., GotTs, N. M. \& LAW, A. N. R. (2001): «Imitative and nonimitative strategies in a land use simulation.» Cybernetics \& Systems 32(1-2), pp. 285-307.

Polhill, J. G., Pignotti, E., Gotts, N. M., Edwards, P. \& Preece, A. (2007): «A Semantic Grid Service for Experimentation with an Agent-Based Model of LandUse Change». Journal of Artificial Societies and Social Simulation 10(2), Artículo 2.

Railsback, S. F., Lytinen, S. L. \& JaCKSON, S. K. (2006): «Agent-based Simulation Platforms: Review and Development Recommendations». Simulation 82(9), pp. 609-623.

REYNOLDS, C. W. (1987) «Flocks, Herds, and Schools: A Distributed Behavioral Model». Computer Graphics, 21(4), pp. 25-34. 
Rodrigues, A. \& Bowers, J. (1996): «System dynamics in project management: A comparative analysis with traditional methods». System Dynamics Review 12(2), pp. 121-139.

Rogers, E. M. (1962): Diffusion of Innovation. New York: Free Press.

SANSORES, C. \& PAVÓN, J. (2005): «Simulación Social Basada en Agentes». Inteligencia Artificial: Revista Iberoamericana de Inteligencia Artificial 9(25), pp. 71-78.

SAnsores, C., PaVón, J. \& Gómez-Sanz, J. (2006): «Visual modeling for complex agent-based simulation systems.» En Sichman, J. S. \& ANTUNES, L. (eds.), MultiAgent-Based Simulation VI, International Workshop, MABS 2005, Utrecht, The Netherlands, July 25, 2005, Revised and Invited Papers, Lecture Notes in Computer Science 3891, pp. 174-189. Berlin Heidelberg: Springer.

SARGENT, R. G. (2003): «Verification and Validation of Simulation Models». En CHICK, S., Sánchez, P. J., Ferrin, D. \& Morrice, D. J. (Eds.) Proceedings of the 2003 Winter Simulation Conference: 37-48. Piscataway, NJ: IEEE.

SCHELling, T. C. (1971): «Dynamic Models of Segregation». Journal of Mathematical Sociology, 1(2), pp. 143-186.

ScHoll, H. (2001): «Agent-based and system dynamics modeling: A call for cross study and joint research». En Proceedings of the 34th Annual Hawaii International Conference on System Sciences (HICSS-34) Vol. 3.

Shan, Y. \& YANG, A. (2008): Applications of Complex Adaptive Systems. IGI Publishing. SQuazzoni, F. (2008): «The Micro-Macro Link in Social Simulation». Sociologica 1/2008, doi: $10.2383 / 26578$.

Sterman, J. D. (2000): Business Dynamics: Systems thinking and modeling for a complex world. McGraw Hill.

Suber, P. (2007): «Formal Systems and Machines: An Isomorphism». Hand-out for «Logical Systems». Earlham College.

TAYLOR, A. J. (1983): «The Verification of Dynamic Simulation Models». Journal of the Operational Research Society, 34(3), pp. 233-242.

TESFATSION, L. (2002): «Agent-based computational economics: growing economies from the bottom up». Artificial life 8(1), pp. 55-82.

- (2003): «Agent-based computational economics: modeling economies as complex adaptive systems». Information Sciences 149(4), pp. 263-269.

Torsun, I. S. (1995): Foundations of Intelligent Knowledge-based Systems. New York: Academic Press.

Vensim. (2008): «Vensim Simulation Software.» from http://www.vensim.com/.

VICSEK, T. (2002): «Complexity: The bigger picture». Nature 418(6894), pp. 131-131.

Walker, D. C., Hill, G., Smallwood, R. H. \& Southgate, J. (2004a): «Agent-based computational modelling of wounded epithelial cell monolayers». IEEE Transactions on Nanobioscience 3, pp. 153-163.

Walker, D. C., Southgate, J., Hill, G., Holcombe, M., Hose, D. R., Wood, S. M., Mac Neil, S. \& Smallwood, R. H. (2004b): «The epitheliome: agent-based modelling of the social behaviour of cells». Biosystems 76(1-3), pp. 89-100.

Wilensky, U. (1999): NetLogo. Evanston, IL Center for Connected Learning and Computer-Based Modeling, Northwestern University. http://ccl.northwestern.edu/ netlogo/.

Windrum, P., Fagiolo, G., \& Moneta, A. (2007): «Empirical Validation of AgentBased Models: Alternatives and Prospects». Journal of Artificial Societies and Social Simulation, 10(2), Artículo 8. 


\title{
RESUMEN
}

Este trabajo compara dos técnicas de modelado de sistemas complejos: la simulación basada en agentes y la dinámica de sistemas. Esta comparación se lleva a cabo dentro del marco general del proceso de modelado científico. Los autores concluyen que la principal diferencia entre las dos metodologías se encuentra en el proceso de abstracción que cada una de ellas realiza para construir el modelo formal a partir del sistema complejo observado. Esta diferencia inicial se extiende a las restantes etapas del proceso de modelado científico. Finalmente, se indican los principales factores y las propiedades generales de un sistema complejo que hacen que una u otra técnica sea más relevante, aunque los autores destacan que, en la mayoría de los casos, modelizar un mismo sistema mediante las dos técnicas es la solución idónea.

\section{PALABRAS CLAVE}

Sistemas complejos, simulación, simulación basada en agentes, dinámica de sistemas, modelado científico.

\begin{abstract}
This paper compares two approaches in complex systems modelling: agentbased modelling and system dynamics. The comparison is undertaken within the general framework of the process of scientific modelling. We argue that the most important difference between the two approaches appears when the modeller builds an abstraction of the target system to develop a formal model from it. This initial difference extends to the following stages of the modelling process, making the two approaches clearly distinctive. Finally, we outline the main general factors that may make one approach more suitable than the other; nevertheless, we also emphasise that modelling the same target system using both techniques seems to be a fruitful and worthy activity.
\end{abstract}

\section{KEY WORDS}

Complex systems, Simulation, Agent-based simulation, System dynamics, Scientific modelling. 